\title{
A supervised learning method for overlapping cells
}

\author{
Pengfei Shen ${ }^{a}$, Jie Yang ${ }^{b}$ \\ Key Laboratory of Fiber Optic Sensing Technology and Information Processing , Ministry of \\ Education, Wuhan University of Technology \\ afeimczjf@163.com, bjieyang509@163.com
}

\begin{abstract}
Keywords: overlapping, non-rigid registration, over-segmentation, template matching Abstract. The clustering phenomenon often appears in histopathology image, some cells overlap or touch together to from a big area. It is necessary to design an effective algorithm to separate the clustering cells into single one. We describe a generic method for segmentation microscopy images based on supervised modeling. The main idea is to use the example input segmentations to learn a statistical model of the shape and texture of the structures to be segmented. The segmentation of the test image can be functioned by maximizing the normalized cross correlation between the model and neighborhoods in the test image, accompanied by a final adjustment that utilizes nonrigid registration. This method can effectively and efficiently solve the overlapping and over-segmentation problem.
\end{abstract}

\section{Introduction}

The automated detection and segmentation of overlapping cells remains one of the most challenging problems in the analysis of microscopic image due to the fundamentally important role of nuclei in the cellular processes and diseases. there exist many approaches to solve the problem, for example, watershed is widely utilized for the segmentation of nuclei image in recent years based on mathematical morphology[1],[2],[3], account of the fact that it has draw great attention for its fast computing and high accuracy in locating the weak edges of adjacent regions, but this method can lead to serious over-segmentation. With regard to other methods, such as a Hybrid Active Contour Model[4], or C-V level set method[5], or spatial graphs built using nuclei as vertices[6], But these means need user intervention, or the effect is not very good when the tissue's color is similar to the nuclei's, or require accurate segmentation of prostate nuclei's as initial step.

In this paper, we utilize a PCA-based method for estimating a mean template from the training data. Segmentation of a subsequent testing image is then performed by searching for the model instance that best fit local region in the test image. The application of this method for segmentation cell image not only solves the problem of overlapping but also overcomes over-segmentation effectively and produces a satisfactory segmentation result.

\section{Material and Methods}

Our method is divided into a training procedure, where a statistical model for the shape and texture for the structures to be segmented is built, and a testing procedure that can segment a given color image.

Training: estimating a mean template. The main work of the training stage is to creating a template model by learning the shape variations[3], as well as mean texture from the image of training data. Each sample just contains a single cell. We first select an arbitrary image from the $\mathrm{N}$ training set images as the initial template. We then register this template to each image in the training set, producing $\mathrm{N}$ non-rigid transformations, and we chose the multi-channel normalized cross correlation(NCC) as the optimization criterion and include coordinate inversions in the optimization procedure. After the rigid registration, we denote these $\mathrm{N}$ rigidly aligned sub-windows as $\mathrm{I}_{1}, \ldots, I_{N}$ from now on, and use them to estimate a template that will represent the shape and texture of these set. In this work we choose the procedure outline in Heitz et al.[2]. The procedure depends on the computation of a non-rigid map that aligns two sample images $I_{i}$ and $I_{\mathrm{j}}$, via $I_{i}\left(f_{i}(x)\right) \approx I_{j}(x)$, with $\mathrm{x}$ an input coordinate in the image grid 
$\Omega$, and $\mathrm{f}_{\mathrm{i}}(\mathrm{x}): \Omega \rightarrow R^{2}$. In our work, we designed a non-registration algorithm to achieve this goal. Let $T(x)$ and $S(x)$ be the two images to be matched, the non-rigid registration algorithm aims to find a warping map $\mu(x)$ that satisfies $T(x) \approx S_{\mu}(x)=S(x-\mu(x))$ via maximizing the square of the multi-channel NCC cost function:

$$
\Phi(\mu)=\frac{1}{N_{c h}} \sum_{j=1}^{N_{c h}}\left(\frac{\left\langle S_{\mu}^{j}, T^{j}\right\rangle}{\left\|S_{\mu}^{j}\right\|^{2}\left\|T^{j}\right\|^{2}}\right)^{2}
$$

Where $N_{c h}$ is the number of channel and $\|T\|^{2}=\langle T, T\rangle=\sum T(x) r(x)$. In this work, equation (1) is maximized via steepest gradient ascent.

Given the ability to non-rigidly align two nuclei image , the template estimation procedure consists of choosing the register image from the data set and denoting it $I_{0}^{k}(x)$. Then, starting with the iteration $\mathrm{k}=1$ :

1. Non-rigidly register $I_{0}^{k}$ to each image $I_{i}$ in data set, $\mathrm{i}=1,2,3, \ldots, \mathrm{N}$ suchthat $I_{i}\left(f_{i}(x)\right) \approx I_{0}^{k}(x)$.

2. Calculate a temporary average shape template $\Psi^{k}(x)=I_{0}^{k}\left(f^{-1}(x)\right)$, with $\bar{f}=\frac{1}{N} \sum_{i=1}^{N} f_{i}$ and $f^{-1}$ the inverse of the transformation function $f$

3. Compute the average texture on the same average shape template above by first registering each data set image to $\Psi^{k}(x) \quad\left(I_{i}\left(f_{i}^{\prime}(x)\right) \approx \Psi^{k}(x)\right)$ and update the template via $I_{0}^{k+1}(x)=\frac{1}{N} \sum_{i=1}^{N} I_{i}\left(f_{i}^{\prime}(x)\right)$.

4. Computer error $e=\left\|I_{0}^{k+1}-I_{0}^{k}\right\|$ (sum of square errors). If $\mathrm{e}<\varepsilon$ stop ,otherwise set $\mathrm{K}=\mathrm{K}+1$ and go to step 1.

The end result is an image $I_{0}(x)$ that represents an average template(shape and texture), as well as a set of spatial transformations that map each image in data set to the final template via $I_{i}\left(f(x)_{i}\right) \approx I_{0}(x), \mathrm{i}=1,2, \ldots, \mathrm{N}$. We next apply the principal component analysis(PCA) technology to derive a statistical model for the possible variations in the shape of the sample nuclei. We encode spatial transformation $f_{i}(x)$ as a vector of displacements via $v_{i}=\left[f_{i}(x), \ldots, f_{i}(x)\right]$, with $\mathrm{L}$ the number of pixels in each image. Thus, the mean and the covariance of the set of spatial displacements $v_{1}, v_{2}, \ldots, v_{N}$ are:

$$
\begin{aligned}
& \bar{V}=\frac{1}{N} \sum_{i=1}^{N} V_{i} \\
& C=\frac{1}{N} \sum_{i=1}^{N}\left(V_{i}-\bar{V}\right)\left(V_{i}-\bar{V}\right)^{T}
\end{aligned}
$$

Using the PCA method, the principal deformation modes are given by the eigenvectors $q_{p}, \mathrm{p}=1,2$, $3, \ldots$ of the covariance matrix $C$ satisfying $C q_{p}=\lambda_{p} q_{p}$. A statistical model for the variations in the shape is obtained by remaining the top eigenvalues and eigenvectors corresponding to $95 \%$ of the variance in the dataset. This means that the number of eigenvector used in each segmentation task will 
depend on how much variability is present in the training set. The model can be evaluated by choosing an eigenvector and calculating $V_{p, b_{p}}=\bar{V}+b_{p} q_{p}$, where $b_{p}$ is a mode coefficient. The corresponding template is obtained by reassembling $V_{p, b_{p}}$ into a corresponding spatial transformation $f_{p, b_{p}}$, and computing $I_{0}\left(f_{p, b_{p}}^{-1}(x)\right)$. We evaluate the model with $b_{p}$ from $-5 \sqrt{\lambda_{p}}$ to $5 \sqrt{\lambda_{p}}$ in intervals of $\sqrt{\lambda_{p}}$. The result of this operator is a set of images obtained by deforming the mean template and representing nuclei configurations likely to be encouraged in data to be segmented.

Segmentation color microscopy images. the segmentation is based on the idea of maximizing the NCC between the statistical model for a given data set and local regions in the test image to be segmented[6],[7]. In the first step, we calculate the multi-channel NCC of the input image[8],[9], here denoted as $J^{i}(x)$, with $\mathrm{i}=1,2,3, \ldots, N_{c h}$, against each filter $W_{p}^{j}(x), \mathrm{p}=1,2,3, \ldots, \mathrm{K}$ in the detection filter bank, and get the response at the pixel $\mathrm{u}$, for example, as:

$$
\gamma_{p}(u)=\frac{1}{N_{c h}} \sum_{j=1}^{N_{c h}} \frac{\sum_{x} J^{j}(x) W_{p}^{j}(x-u)}{\bar{J}^{j}(u) \bar{W}_{p}^{j}}
$$

Where $\bar{W}_{p}^{i}=\left(\sum_{x}\left(W_{p}^{i}(x)\right)^{2}\right)^{\frac{1}{2}}$ and $\bar{J}^{i}(u)=\left(\sum_{x \in \Omega_{u}} J^{i}(u)^{2}\right)^{\frac{1}{2}}$. A detection probability image M can be computed by keeping the maximum response at the pixel $\mathrm{u}$ as: $M(u)=\max _{p} \gamma_{p}(u)$. Potential guesses are identified by finding the pixel location in $\mathrm{M}$ which are greater than a threshold $\mu$.To that end, the threshold detection image $M$ is searched for its highest response, then the second highest which is at least a distance $\gamma$ from the first, the third highest which are at least distance $\gamma$ from the previous two, and so on. The process is repeated until all pixels in the threshold detection image $\mathrm{M}$ have been investigated. We note that each detection pixel in $\mathrm{M}$ also has its associated best match from the detection filter bank. Consequently, the output provides not only the locations of nuclei but also a rough guess for its shape from the binary mask of its best matching template.

\section{Result and Conclusion}

we apply our method to segment color microscopy images of nuclei obtained using histopathology techniques. An important feature of our template matching approach is that it is capable of segmenting the overlapping nuclei without any difficulties. An example in Fig.1 shows the result of our method and the method based on watershed. The result of our method is shown in Figuhich is the disadvantage of the method of watershed. Fig.1(a) is the original image, and the result of watershed is shown in figure 1(b). The Fig.1(c) is the result of our method. From the figure, we can know that our approach can solve the overlapping and at the same time can effectively overcome the over-segmentation .

we describe a method for segmenting cell nuclei from several different modalities of image based on supervised learning and template matching. An important feature of our template matching is that it is captable of segmentation touching nuclei without difficulties and at the same time, it can effectively figure out the over-segmentation problem. The mehod is suitable for a variety of imaging experiments given that it contains a training step that adapts the statictical model consists of building a statistical model for the texture and shape variations of the nuclei from the input of data set, and then segmeting arbitrary images by finding the instance in the model that best matches, in the sense of the NCC, local region in the input images. Our method motivation was to design a method for segmenting nuclei from microscopy images of arbitrary types(scalar, color, fluorescence, different staining,etc). Account of the fact that the train of the supervised learing always expend too much time, our method maybe not be not very fast with regard to time, even though the effct is very well. 


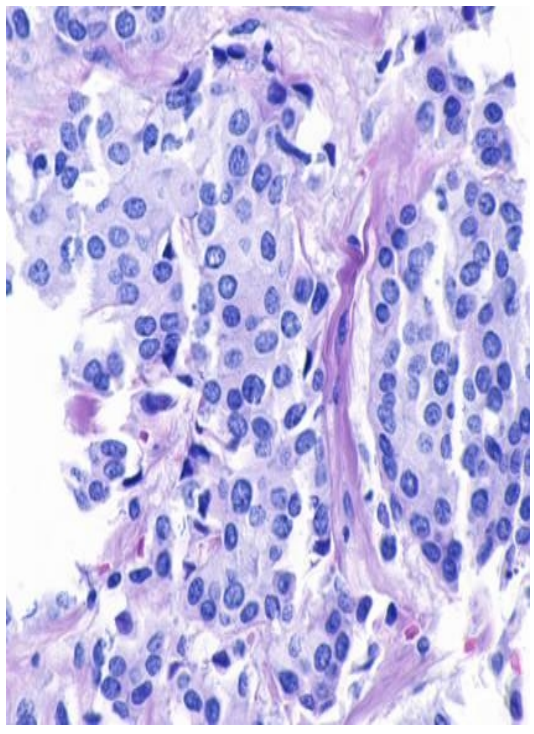

(a)

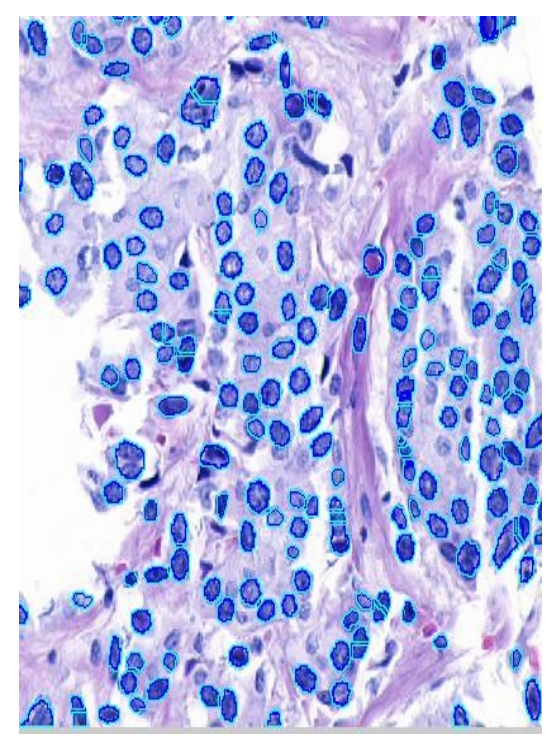

(b)

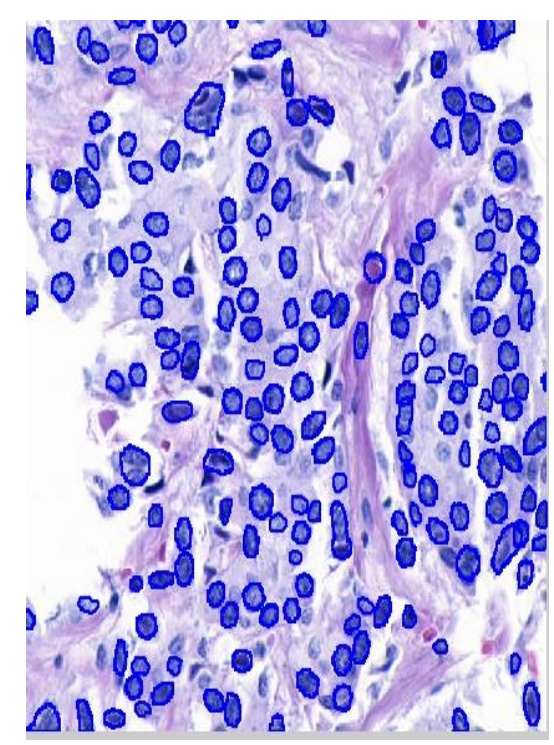

(c)

Figure1. results on histopathology nuclei images (a) original image (b) The over-segmentation image by watershed method (c) this paper's result

\section{Acknowledgement}

This work is supported by the foundation of Independent Innovation research in colleges and universities (2014-zy-120).

\section{References}

[1] L.P. Coelho, A. Shariff, and R.F. Murphy, "Nuclear segmentation in microscope cell images: a hand-segmented dataset and comparison of algorithms," in Biomedical Imaging: From Nano to Macro, 2009.ISBI'09. IEEE International Symposium on. IEEE, 2009, pp. 518-521.

[2] C.Cheng,W.Wang, J.A.Ozolek, and G.K.Rohde,"A template matching approach for segmentation cell nuclei from microscopy images," Medical Imaging ,IEEE Transactions on, in preparation.

[3] H Huang, AB Tosun, J Guo, C Chen, W Wang, JA Ozolek, GK Rohde, Cancer diagnosis by nuclear morphometry using spatial information. Pattern Recognition Letters. 2014,42, 115-121.

[4] S. Basu, K. N. Dahl, G. K. Rohde, Localizing and extracting filament distributions from microscopy images, Journal of Microscopy, 2013,vol. 250(1), pp 57-67, PMC3638952.

[5] GK Rohde, Computational Analysis of Cell Images, Cytometry A, 2015,87 (4), 294-295.

[6] F. Eisses, A. Davis, A.B. Tosun, Z.R. Dionise, C. Cheng, J.A. Ozolek, G.K. Rohde and S.Z. Husain, A Computer-Based Automated Algorithm for Assessing Acinar Dropout after Experimental Pancreatitis, PLOS ONE, 2014,9(10), e110220.

[7] JA Ozolek, AB Tosun, W Wang, C Chen, S Basu, H Huang, GK Rohde. Accurate diagnosis of thyroid follicular lesions from nuclear morphology using supervised learning. Medical Image Analysis. 2014,18(5), 772-780. 
[8] S. Xu, L Sun, GK Rohde, Robust efficient estimation of heart rate pulse from video. Biomedical Optics Express 2014,5(4), 1124-1135.

[9] H Huang, AB Tosun, J Guo, C Chen, W Wang, JA Ozolek, GK Rohde, Cancer diagnosis by nuclear morphometry using spatial information. Pattern Recognition Letters. 2014,42, 115-121. 